Research Article

\title{
Energy-Efficient UAV Communication with Multiple GTs Based on Trajectory Optimization
}

\author{
Yu Xu, ${ }^{1}$ Lin Xiao $\left(D,{ }^{1}\right.$ Dingcheng Yang, ${ }^{1}$ Laurie Cuthbert, ${ }^{2}$ and Yapeng Wang ${ }^{3}$ \\ ${ }^{1}$ Information Engineering School, Nanchang University, Nanchang 330031, China \\ ${ }^{2}$ Information Systems Research Centre, Macao Polytechnic Institute, Rua de Luis Gonzaga Gomes, Macao SAR, China \\ ${ }^{3}$ MPI-QMUL Information Systems Research Centre, Macao Polytechnic Institute, Macao SAR, China
}

Correspondence should be addressed to Lin Xiao; xiaolin@ncu.edu.cn

Received 26 November 2017; Accepted 18 February 2018; Published 10 April 2018

Academic Editor: Jose F. Monserrat

Copyright (C) $2018 \mathrm{Yu} \mathrm{Xu} \mathrm{et} \mathrm{al.} \mathrm{This} \mathrm{is} \mathrm{an} \mathrm{open} \mathrm{access} \mathrm{article} \mathrm{distributed} \mathrm{under} \mathrm{the} \mathrm{Creative} \mathrm{Commons} \mathrm{Attribution} \mathrm{License,} \mathrm{which}$ permits unrestricted use, distribution, and reproduction in any medium, provided the original work is properly cited.

\begin{abstract}
Wireless communications with unmanned aerial vehicles (UAVs) is a promising technology offering potential high mobility and low cost. This paper studies a UAV-enabled communication system, in which a fixed-wing UAV is deployed to collect information from a group of distributed ground terminals (GTs). Considering the requirements for quality of service (QoS) (i.e., the throughput of each GT is above a given threshold) and GT scheduling, we maximize the energy efficiency (EE) of the UAV in bits/Joule by optimizing the UAV's flight trajectory. In this paper, a mixed integer nonconvex optimization problem is formulated. As that is difficult to solve, we divide the formulated problem into two subproblems and apply standard linear programming (LP) and successive convex optimization techniques. We further propose an efficient iterative algorithm that jointly optimizes GT scheduling and the UAV's trajectory. Moreover, we set two special cases as benchmarks to measure the performance of the proposed design. The numerical results show that our proposed design achieves much better performance than the other two benchmark designs.
\end{abstract}

\section{Introduction}

Unmanned aerial vehicles (UAVs) recently are attracting significant attention in many fields: they can be applied in many different scenarios, including surveillance, monitoring, mobile relays, and data collection [1]. In general, UAVs can provide line-of-sight (LoS) links and thus offer good link capacity. Due to their potential mobility, flexible deployment, and low cost, UAVs are available for many operations and applications, such as precision agriculture [2], search and rescue [3], and timely environment monitoring and disaster warning [4-6]. Furthermore, UAVs can be used as mobile relays to extend the capacity and coverage of networks [7]. Meanwhile, with increasing popularity in the field of information technology (IT), both Facebook Aquila Drone [8] and Google Loon Project [9] aim to provide ubiquitous internet access for users in remote locations by using UAVs. In addition, UAVs can be deployed as aerial base stations (BSs) for ground terminals (GTs) as they are flexibly reconfigured
[10-12]. UAVs therefore provide aerial platforms that can be widely applied in wireless communication systems, as they can provide the terrestrial-aerial communication service for terrestrial users in regions lacking terrestrial infrastructures or under overload conditions $[13,14]$.

Nevertheless, UAV communication systems still face many critical challenges [1]. One of these is the limited battery capacity so that the UAVs have to land for recharging, which severely restricts the endurance of UAVs. As a result, the energy efficiency (EE) in bits/Joule is an important performance metric in UAV wireless communication [15]. Note that, unlike conventional terrestrial systems, the UAV needs to consume propulsion power to remain aloft in addition to the power required for the communications [16]. Therefore, having an energy-efficient trajectory design for UAV communication systems is of paramount importance. Hence, the requirement is to guarantee high-rate communication for the network, with low propulsion energy consumption. 
Moreover, a UAV communication system with multiple GTs unavoidably leads to a higher performance requirement for the UAV and adds many key issues (e.g., user scheduling, user fairness, and access delay) that are challenging to solve. Note that energy-efficient designs for UAV communication systems are significantly different from traditional cellular settings:

(i) Saving power and cost by energy-efficient designs for UAV communication systems is more critical than that for terrestrial communication systems because of the limited on-board energy.

(ii) In addition to the communication-related power consumption, the propulsion power requirement for UAVs leads to much higher overall consumption.

We take both of these factors into account in this paper for energy-efficient designs for UAV communications.

In previous work $[12,17,18]$, the maximum coverage for GTs is studied by optimizing the deployment of UAVs. However, this work does not focus on the issue of the UAV's energy efficiency. The work in [19] studies the energy-efficient $3 \mathrm{D}$ placement of a UAV-BS to achieve maximum coverage for users with the minimum required transmit power, but it does not consider the information bit requirement of all users. In [20], the UAV is deployed in a circular trajectory to assist communication and an energy-efficient design is considered, but it does not take multiple GTs into consideration. The authors in [15] studied energy-efficient communication by optimizing the UAV's trajectory: a mathematical model for the propulsion energy consumption of a fixed-wing UAV was developed, but they discuss neither the condition of multiple GTs nor QoS requirements for GTs.

This paper aims to study the EE maximization design for a UAV communication system with a group of GTs over a finite time period. In this system, a UAV is dispatched to collect the uploading data from GTs. We assume that these GTs upload data to the UAV using time-division multiple access (TDMA) that can efficiently avoid the cochannel interference enabling the frequency band to be shared. In fact, we discuss a new system model jointly considering QoS requirements and GT scheduling. Our objective is to maximize the UAV's energy efficiency by optimizing the trajectory. In addition, we set two special cases as benchmarks for comparison as well as to indicate the optimal performance of the objective problem. For the multiple GT system, it is important to note how the UAV chooses to communicate with which GT in each time slot. Hence, the GT scheduling is crucial to improve the UAV's EE. Intuitively, the UAV needs to be close to the associated GT that is transmitting information to the UAV for a better UAV-GT channel [21], but the required propulsion energy of the UAV is unfortunately likely to be larger. In general, the solution to the problem of the UAV's EE maximization can be derived at an optimal balance point between throughput maximization and energy consumption minimization.

The rest of this paper is organized as follows: first, Section 2 presents the system model and the problem formulation. Then, Section 3 proposes an effective alternative iteration algorithm. The numerical results are presented in Section 4 to

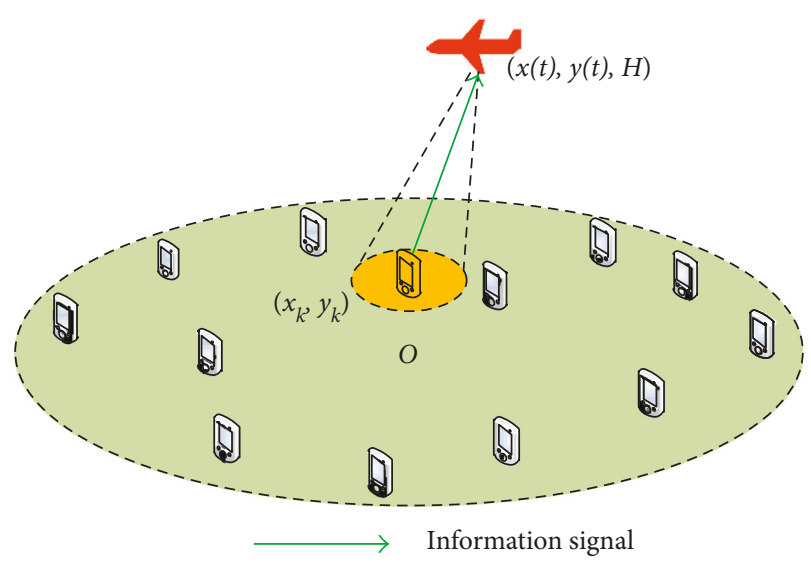

FIGURE 1: A UAV wireless communication system with multiple GTs.

demonstrate our proposed design. Finally, our main conclusions of the paper are summarized in Section 5.

\section{System Model and Problem Formulation}

2.1. System Model. As shown in Figure 1, we consider a UAV wireless communication system with $N$ GTs denoted as the set $\mathscr{N}=\{1,2, \ldots, N\}$. Without loss of generality, we consider a 3D Cartesian coordinate system. We assume that the group of GTs is distributed on a given circular geographical area with the geometrical center $[0,0]^{T}$. The horizon coordinate of the GT $k$ is predetermined and fixed at $\left\{\mathbf{w}_{k}=\left[x_{k}, y_{k}\right]^{T}\right\}_{k \in \mathcal{N}^{\prime}}$ $\mathbf{w}_{k} \in \mathbb{R}^{2 \times 1}$. In the system, the UAV is dispatched to collect the uplink data from GTs during the time horizon $T$. We assume that the altitude of the UAV is fixed at $H$ meters that correspond to the minimum altitude to avoid collision. Consequently, the UAV trajectory can be denoted by $[x(t), y(t), H]^{T}, 0 \leq t \leq T$, with $x(t)$ and $y(t)$ denoting the time-varying $x$ - and $y$-coordinates projected on the horizontal plane. Therefore, the UAV's projected position can be denoted by $\mathbf{q}(t)=[x(t), y(t)]^{T}$, where $0 \leq t \leq T$. For ease of formulation of the problem, we introduce a sufficiently small time step $\delta_{\mathrm{t}}$ and then discretize the period time into $M$ equal time slots with the step size $\delta_{t}$, indexed by $n=1, \ldots, M$. We assume that the trajectory of the UAV satisfies the following constraint:

$$
\begin{aligned}
& \mathbf{q}[1]=\mathbf{q}[M], \\
& \mathbf{v}[1]=\mathbf{v}[M],
\end{aligned}
$$

where $\mathbf{v}[n]$ denotes the UAV's velocity in time slot $n$. From [15], we have the linear relationships as follows:

$$
\begin{aligned}
\mathbf{q}[n+1] & =\mathbf{q}[n]+\mathbf{v}[n] \delta_{\mathrm{t}}+\frac{1}{2} \mathbf{a}[n] \delta_{\mathrm{t}}^{2}, \\
\mathbf{v}[n+1] & =\mathbf{v}[n]+\mathbf{a}[n] \delta_{\mathrm{t}}, \quad n=1,2, \ldots, M-1,
\end{aligned}
$$

where $\mathbf{a}[n]$ denotes the UAV's acceleration during time slot $n$. Thus, in any time slot, we can express the time-varying distance between the UAV and the $k$ th GT as

$$
d_{k}[n]=\sqrt{\left\|\mathbf{q}[n]-w_{k}\right\|^{2}+H^{2}}, \quad k \in \mathcal{N} .
$$

Furthermore, we assume that the other GTs do not cause any interference on the current communication channel between the 
UAV and the $k$ th GT. Moreover, we consider that each UAV-GT channel follows a line-of-sight (LoS) link. Actually, practical UAV-GT channels can be well approximated by the LoS model. We also assume that the Doppler effect due to the UAV's mobility is perfectly compensated [22]. Therefore, the channel power gain from the $k$ th GT to the UAV conforms to the freespace path loss model, which can be expressed as

$$
h_{k}[n]=\beta_{0} d_{k}^{-2}[n]=\frac{\beta_{0}}{\left\|\mathbf{q}[n]-w_{k}\right\|^{2}+H^{2}}, \quad k \in \mathcal{N},
$$

where $\beta_{0}$ denotes the channel power at the reference distance $d_{0}=1$ meter. We define a binary variable $\alpha_{k}[n]$ that represents the communication scheduling factor for GTs; namely, it indicates whether or not the UAV collects data from the GT $k$ during time slot $n$. If $\alpha_{k}[n]=1$, it shows that the UAV communicates with the GT $k$ in time slot $n$. Otherwise, we let $\alpha_{k}[n]=0$. Denoted by $P$, the transmission power of each GT in per time slot is constant. The instantaneous channel capacity between the UAV and the GT $k$ in bits/second/Hz (bps/Hz) can be written as

$$
\begin{aligned}
r_{k}[n] & =\alpha_{k}[n] \log _{2}\left(1+\frac{h_{k}[n] P}{\sigma^{2}}\right) \\
& =\alpha_{k}[n] \log _{2}\left(1+\frac{\gamma_{0} P}{\left\|\mathbf{q}[n]-\mathbf{w}_{k}\right\|^{2}+H^{2}}\right), \quad k \in \mathcal{N},
\end{aligned}
$$

where $\sigma^{2}$ is the white Gaussian noise power at each GT receiver and $\gamma_{0}=\beta_{0} / \sigma^{2}$ denotes the reference-received signal-to-noise ratio (SNR) at $d_{0}=1$ meter. We assume that the communication bandwidth for the UAV is $B$, and each GT can share the whole frequency band using time-division multiple access (TDMA). Therefore, the throughput of the GT $k$ in the period time $T$ is given by

$$
\begin{aligned}
R_{k} & =\delta_{t} B \sum_{n=1}^{M} r_{k}[n] \\
& =\delta_{t} B \sum_{n=1}^{M} \alpha_{k}[n] \log _{2}\left(1+\frac{P \gamma_{0}}{\left\|\mathbf{q}[n]-\mathbf{w}_{k}\right\|^{2}+H^{2}}\right), \quad k \in \mathscr{N} .
\end{aligned}
$$

Therefore, the total amount of information bits transmitted from all GTs to the UAV over the horizon time $T$ can be expressed as

$$
\begin{aligned}
& R\left(\{\mathbf{q}[n]\},\left\{\alpha_{k}[n]\right\}\right) \\
& \quad=\sum_{k=1}^{N} R_{k} \\
& \quad=\delta_{t} B \sum_{k=1}^{N} \sum_{n=1}^{M} \alpha_{k}[n] \log _{2}\left(1+\frac{P \gamma_{0}}{\left\|\mathbf{q}[n]-\mathbf{w}_{k}\right\|^{2}+H^{2}}\right), \\
& k \in \mathcal{N} .
\end{aligned}
$$

In our proposed system, the energy consumption of the UAV consists of two parts. The first is the communicationrelated energy consumption and the second is the propulsion energy consumption, which ensures the UAV remains in the air, supporting its mobility. In practice, the communicationrelated energy is small compared with the propulsion energy. Thus, we ignore the communication-related energy in this paper. From [15], the energy consumption of a fixed-wing UAV during $T$ can be expressed as

$$
E(\{\mathbf{q}[n]\})=\delta_{\mathrm{t}} \sum_{n=1}^{M}\left[c_{1}\|\mathbf{v}[n]\|^{3}+\frac{c_{2}}{\|\mathbf{v}[n]\|}\left(1+\frac{\|\mathbf{a}[n]\|^{2}-\left(\left(\mathbf{a}^{T}[n] \mathbf{v}[n]\right)^{2} /\|\mathbf{v}[n]\|^{2}\right)}{g^{2}}\right)\right]+\Delta_{k}
$$

where $c_{1}$ and $c_{2}$ are two constant parameters related to aerodynamics. The second term $\Delta_{k}$ denotes the UAV's kinetic energy, whose value only depends on the initial and final speed, that is, $\Delta_{k}=(1 / 2) m\left(\|\mathbf{v}[M]\|^{2}-\|\mathbf{v}[1]\|^{2}\right)$. Obviously, under our hypothesis in (2), we can obtain $\Delta_{k}=0$.
2.2. Problem Formulation. In this paper, our objective is to maximize the UAV's energy efficiency by jointly optimizing GT scheduling and the UAV's trajectory. With (8), (9), and some constrains like (1) and (2), the optimization problem can be formulated as

$$
\begin{aligned}
& \max _{\{\mathbf{q}[n], \mathbf{v}[n], \mathbf{a}[n]\},\left\{\alpha_{k}[n]\right\}} \frac{B \sum_{k=1}^{N} \sum_{n=1}^{M} \alpha_{k}[n] \log _{2}\left(1+\left(P \gamma_{0} /\left(\left\|\mathbf{q}[n]-\mathbf{w}_{k}\right\|^{2}+H^{2}\right)\right)\right)}{\sum_{n=1}^{M}\left(c_{1}\|\mathbf{v}[n]\|^{3}+\left(c_{2} /\|\mathbf{v}[n]\|\right)+\left(c_{2}\|\mathbf{a}[n]\|^{2} / g^{2}\|\mathbf{v}[n]\|\right)\right)}, \\
& \text { s.t. } \mathbf{q}[n+1]=\mathbf{q}[n]+\mathbf{v}[n] \cdot \delta_{\mathrm{t}}+\frac{1}{2} \mathbf{a}[n] \cdot \delta_{\mathrm{t}}^{2}, \quad n=1,2, \ldots, M-1,
\end{aligned}
$$




$$
\begin{aligned}
& \mathbf{v}[n+1]=\mathbf{v}[n]+\mathbf{a}[n] \cdot \delta_{\mathrm{t}}, \quad n=1,2, \ldots, M-1, \\
& \mathbf{q}[1]=\mathbf{q}[M], \\
& \mathbf{v}[1]=\mathbf{v}[M], \\
&\|\mathbf{v}[n]\| \leq V_{\max }, \quad \forall n, \\
&\|\mathbf{a}[n]\| \leq a_{\max }, \quad \forall n, \\
& \sum_{k=1}^{N} \alpha_{k}[n] \leq 1, \quad \forall n, \\
& \alpha_{k}[n] \in\{0,1\}, \quad \forall k, n, \quad(10 \mathrm{~b}) \\
& B \delta_{\mathrm{t}} \sum_{n=1}^{M} \alpha_{k}[n] \log _{2}\left(1+\frac{P \gamma_{0}}{\left\|\mathbf{q}[n]-\mathbf{w}_{k}\right\|^{2}+H^{2}}\right) \geq Q_{\mathrm{m}}, \quad k \in \mathcal{N},
\end{aligned}
$$

where the constraints (10c) and (10d) indicate that the UAV needs to get back to the initial location after the horizon time $T$ with the same initial and final velocity, which is opportune for the UAV to take the next cycle flight via the same trajectory. In the constraints (10e) and (10f), $V_{\max }$ and $a_{\max }$ denote the maximum velocity and acceleration that the UAV can achieve. The constraints $(10 \mathrm{~g})$ and $(10 \mathrm{~h})$ represent that the UAV can communicate with one GT at most during any time slot. The last constraint (10i) is the QoS requirement for all GTs, and $Q_{m}$ indicates the minimum amount of uploading data for each GT in the period time $T$.

Note that problem (10) is difficult to solve due to two main reasons:

(i) Firstly, the constraints (10g) and (10h) involve integer constraints. Moreover, the constraint (10i) is nonconvex.

(ii) The objective function of (10) is nonconcave. As a result, problem (10) is a mixed-integer nonconvex problem that cannot be solved directly by using conventional convex optimization techniques in general.

Before discussing the solution to (10), we need to introduce two special cases, which can be used as benchmarks in this paper.

2.3. Special Case I: Average Time Allocation Tactics. In this special case, an approach using average time allocation tactics is presented: each GT always is allocated equivalent communication time during the horizon time $T$. In this case, we can use a vector $\alpha_{k}^{\text {Ave }}[n]$ to denote the GT scheduling. Similarly, $\alpha_{k}^{\text {Ave }}[n]=1$ denotes that the UAV collects data from the $k$ th GT in slot $n$. Otherwise, $\alpha_{k}^{\text {Ave }}[n]=0$. There always is $\sum_{n=1}^{M} \alpha_{k}^{\text {Ave }}[n]=M / N, \forall k \in \mathcal{N}$, where $M / N$ is assumed to be an integer. In this scenario, the problem of this case can be formulated as

$$
\begin{aligned}
& \max _{\left\{\mathbf{q}[n], \mathbf{v}[n], \mathbf{a}[n], \alpha_{k}^{\text {Ave }}[n]\right\}} \frac{B \sum_{k=1}^{N} \sum_{n=1}^{M} \alpha_{k}^{\mathrm{Ave}}[n] \log _{2}\left(1+\left(P \gamma_{0} /\left(\left\|\mathbf{q}[n]-\mathbf{w}_{k}\right\|^{2}+H^{2}\right)\right)\right)}{\sum_{n=1}^{M}\left(c_{1}\|\mathbf{v}[n]\|^{3}+\left(c_{2} /\|\mathbf{v}[n]\|\right)+\left(c_{2}\|\mathbf{a}[n]\|^{2} / g^{2}\|\mathbf{v}[n]\|\right)\right)}, \\
& \text { s.t. (10a)-(10f). } \\
& \quad B \delta_{\mathrm{t}} \sum_{n=1}^{M} \alpha_{k}^{\text {Ave }}[n] \log _{2}\left(1+\frac{P \gamma_{0}}{\left\|\mathbf{q}[n]-\mathbf{w}_{k}\right\|^{2}+H^{2}}\right) \geq Q_{\mathrm{m}}, \quad k \in \mathcal{N} .
\end{aligned}
$$

Compared to problem (10), except the constraint (11a), all constraints in problem (11) are convex. Nevertheless, the objective function of (11) is still nonconcave, and we need to convert it into a new form consisting of a concave numerator and a convex denominator that can be solved efficiently in Section 3 .

2.4. Special Case II: Maximum EE without QoS. In this part, we discuss the other special case, in which we just consider the optimal GT scheduling and UAV trajectory design rather than taking into account the QoS requirements, which means that this design has a higher probability of achieving a larger total system rate in each iteration but may result in the throughputs of some GTs being under the threshold. For this case, it can also be considered as a special case of problem (10), and this case can be formulated as

$$
\begin{aligned}
& \max _{\left\{\mathbf{q}[n], \mathbf{v}[n], \mathbf{a}[n], \alpha_{k}[n]\right\}} \frac{B \sum_{k=1}^{N} \sum_{n=1}^{M} \alpha_{k}[n] \log _{2}\left(1+\left(P \gamma_{0} /\left(\left\|\mathbf{q}[n]-\mathbf{w}_{k}\right\|^{2}+H^{2}\right)\right)\right)}{\sum_{n=1}^{M}\left(c_{1}\|\mathbf{v}[n]\|^{3}+\left(c_{2} /\|\mathbf{v}[n]\|\right)+\left(c_{2}\|\mathbf{a}[n]\|^{2} / g^{2}\|\mathbf{v}[n]\|\right)\right)}, \\
& \text { s.t. (10a)-(10h). }
\end{aligned}
$$


As problem (12) is a special case of problem (10), it can also be efficiently solved with the optimization technique proposed in Section 3.

\section{Jointly Optimal Communication Scheduling and Trajectory Design}

In this section, we propose a joint optimization method by applying the standard LP and successive convex optimization techniques to handle problem (10). We first split problem (10) into two subproblems. For the first subproblem, we can obtain $\left\{\alpha_{k}^{j+1}[n]\right\}$ by optimizing the GT scheduling factor $\left\{\alpha_{k}[n]\right\}$ with the given UAV's trajectory $\left\{\mathbf{q}_{j}[n]\right\}$ in the $(j+1)$ th iteration. As to the second subproblem, we fix the GT scheduling $\left\{\alpha_{k}^{j+1}[n]\right\}$ and then optimize the trajectory of the UAV. Following this iterative method, the optimal energyefficient trajectory of the UAV eventually can be obtained.

3.1. Optimal GT Scheduling with Fixed Trajectory. Considering that this subproblem is a $0-1$ integer programming with the given UAV's trajectory $\left\{\mathbf{q}_{j}[n]\right\}$, we relax the binary variables $\left\{\alpha_{k}[n]\right\}$ in (10h) into continuous variables. Thus, we have the following optimization problem:

$$
\max _{\left\{\alpha_{k}[n]\right\}} \sum_{k=1}^{N} \sum_{n=1}^{M} \alpha_{k}[n] \log _{2}\left(1+\frac{P \gamma_{0}}{\left\|\mathbf{q}_{j}[n]-\mathbf{w}_{k}\right\|^{2}+H^{2}}\right),
$$

s.t. $(10 \mathrm{~g})$,

$$
0 \leq \alpha_{k}[n] \leq 1, \quad \forall n, k
$$

$B \delta_{\mathrm{t}} \sum_{n=1}^{M} \alpha_{k}[n] \log _{2}\left(1+\frac{P \gamma_{0}}{\left\|\mathbf{q}_{j}[n]-\mathbf{w}_{k}\right\|^{2}+H^{2}}\right) \geq Q_{\mathrm{m}}, \quad k \in \mathcal{N}$.

Obviously, problem (13) is a standard linear programming (LP) problem, so it can be solved easily and efficiently using optimization tools. By solving problem (13), we can achieve the optimal GT scheduling factor $\left\{\alpha_{k}^{j+1}[n]\right\}$, and then, $\left\{\alpha_{k}^{j+1}[n]\right\}$ serves as the input for problem (14) in the next subsection.

3.2. Optimal UAV's Trajectory Design with Fixed GT Scheduling. In this subsection, with the given $\left\{\alpha_{k}^{j+1}[n]\right\}$, we need to solve the following subproblem:

$$
\begin{aligned}
& \max _{\{\mathbf{q}[n], \mathbf{v}[n], \mathbf{a}[n]\}} \frac{B \sum_{k=1}^{N} \sum_{n=1}^{M} \alpha_{k}^{j+1}[n] \log _{2}\left(1+\left(P \gamma_{0} /\left(\left\|\mathbf{q}[n]-\mathbf{w}_{k}\right\|^{2}+H^{2}\right)\right)\right)}{\sum_{n=1}^{M}\left(c_{1}\|\mathbf{v}[n]\|^{3}+\left(c_{2} /\|\mathbf{v}[n]\|\right)+\left(c_{2}\|\mathbf{a}[n]\|^{2} / g^{2}\|\mathbf{v}[n]\|\right)\right)}, \\
& \text { s.t. (10a)-(10f), }
\end{aligned}
$$$$
B \delta_{\mathrm{t}} \sum_{n=1}^{M} \alpha_{k}^{j+1}[n] \log _{2}\left(1+\frac{P \gamma_{0}}{\left\|\mathbf{q}[n]-\mathbf{w}_{k}\right\|^{2}+H^{2}}\right) \geq Q_{\mathrm{m}},
$$$$
k \in \mathcal{N}
$$

where $\|\mathbf{v}[n]\|^{2} \geq \kappa_{n}^{2}, \forall n$. Note that this new constraint is nonconvex. We know that $\|\mathbf{v}[n]\|^{2}$ is convex; thus, its firstorder Taylor expansion is the global underestimator. As a result, we adopt the Taylor approximation at the given local point $\left\{\mathbf{v}_{j}[n]\right\}$ and define the lower bound function as follows:

Unfortunately, (14) is not a standard convex problem, but we can find the local optimal solution by applying the sequential convex optimization technique. Hence, we first introduce slack variables $\left\{\kappa_{n}\right\}$ to reform the denominator of the objective function in (14) as

$$
P\left(\{\mathbf{q}[n]\},\left\{\kappa_{n}\right\}\right)=\sum_{n=1}^{M}\left(c_{1}\|\mathbf{v}[n]\|^{3}+\frac{c_{2}}{\kappa_{n}}+\frac{c_{2}\|\mathbf{a}[n]\|^{2}}{g^{2} \kappa_{n}}\right),
$$

$$
\rho_{l b}(\mathbf{v}[n]) \triangleq\left\|\mathbf{v}_{j}[n]\right\|^{2}+2 \mathbf{v}_{j}^{T}[n]\left(\mathbf{v}[n]-\mathbf{v}_{j}[n]\right), \quad \forall n .
$$

It is noteworthy that the numerator of the objective function in (14) is nonconcave with respect to $\{\mathbf{q}[n]\}$. However, if we consider $\left\|\mathbf{q}[n]-\mathbf{w}_{k}\right\|^{2}$ as a whole, the numerator is convex with respect to it. Therefore, with the given local point $\left\{\mathbf{q}_{j}[n]\right\}$ at the $(j+1)$ th iteration, we can obtain the lower bound function of the numerator as follows:

$$
\begin{aligned}
R(\{\mathbf{q}[n]\}) & =B \sum_{k=1}^{N} \sum_{n=1}^{M} \alpha_{k}^{j+1}[n] \log _{2}\left(1+\frac{P \gamma_{0}}{\left\|\mathbf{q}[n]-\mathbf{w}_{k}\right\|^{2}+H^{2}}\right) \\
& \geq B \sum_{k=1}^{N} \sum_{n=1}^{M}\left[\varphi_{j}[n]-\psi_{j}[n]\left(\left\|\mathbf{q}[n]-\mathbf{w}_{k}\right\|^{2}-\left\|\mathbf{q}_{j}[n]-\mathbf{w}_{k}\right\|^{2}\right)\right] \\
& \triangleq R_{l b}(\{\mathbf{q}[n]\}), \quad \forall n,
\end{aligned}
$$




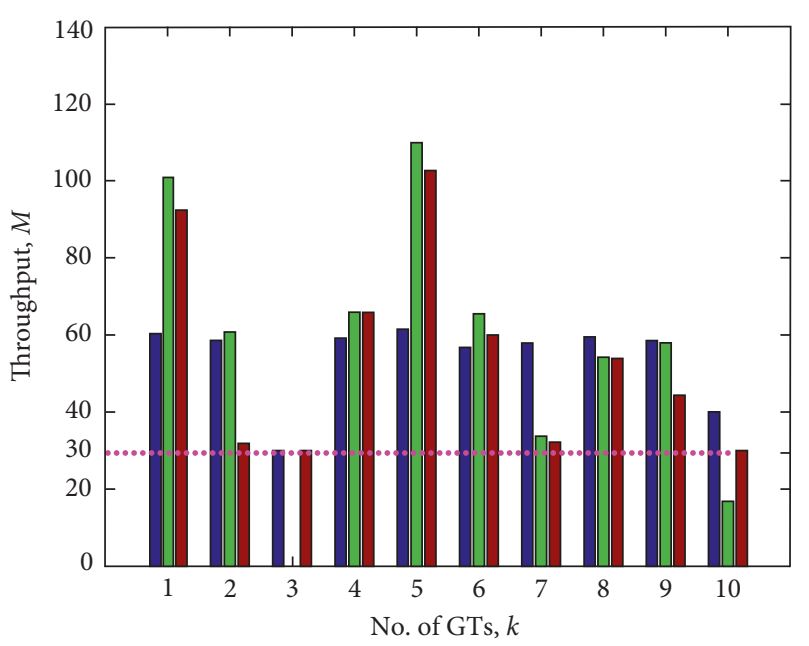

Throughput with average time allocation Throughput of GTs without QoS Throughput of GTs with QoS

(a)

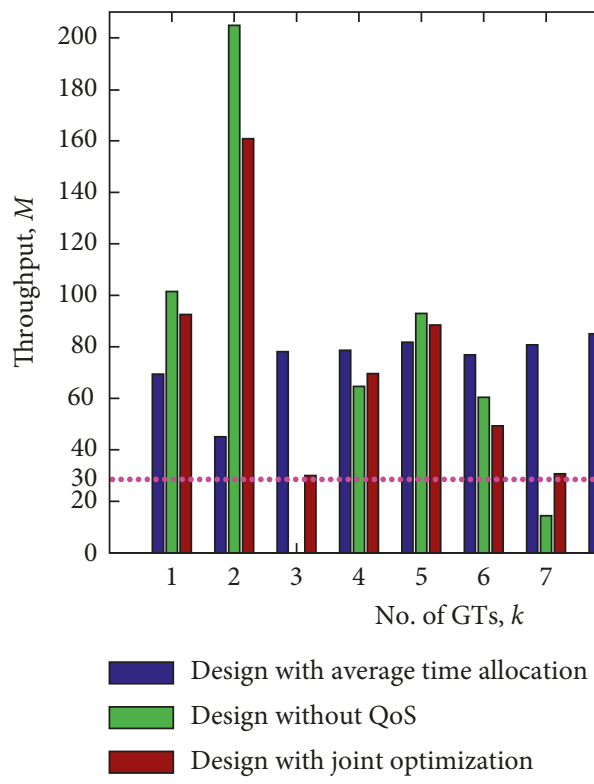

(c)

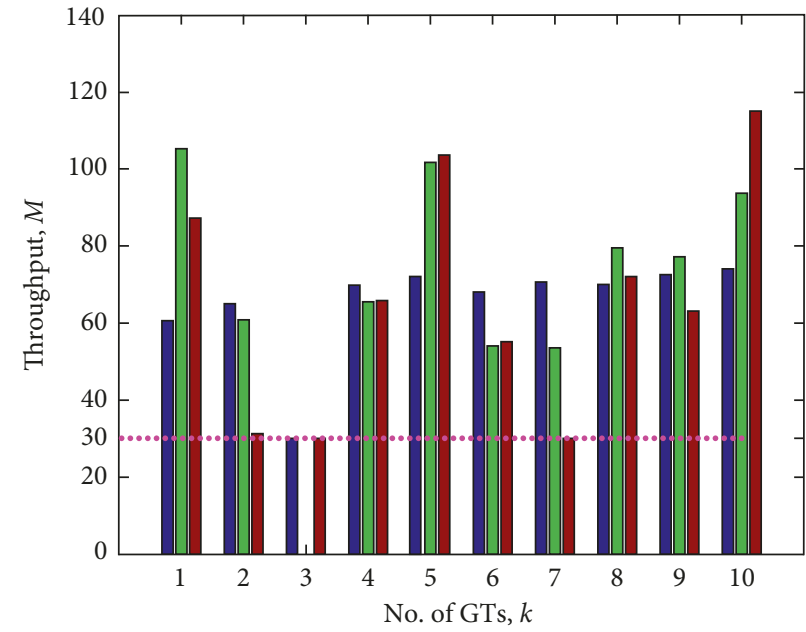

Throughput with average time allocation Throughput of GTs without QoS Throughput of GTs with QoS

(b)

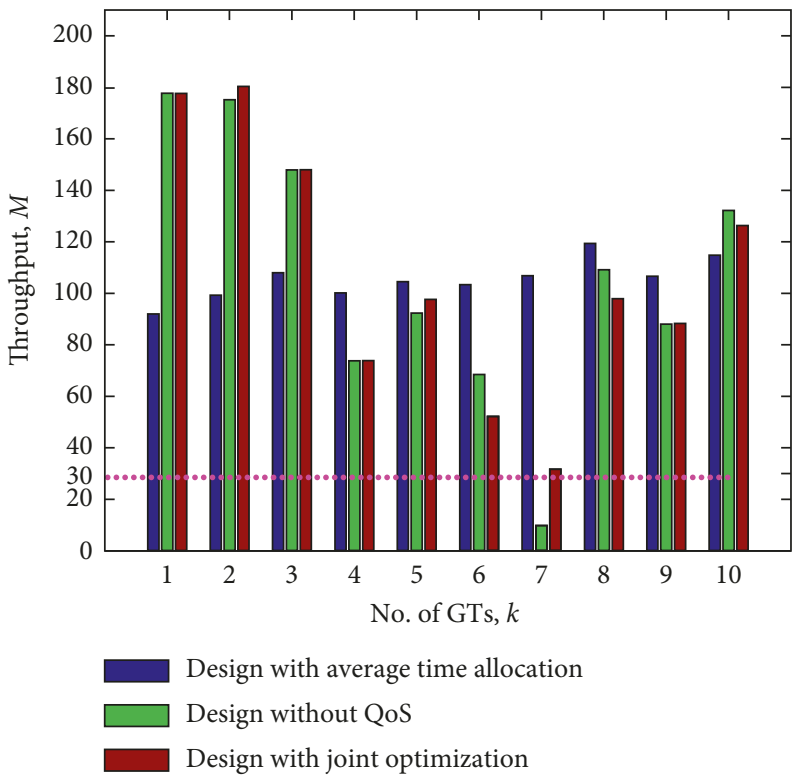

(d)

FIGURE 2: Comparison of the throughput of per GT with different period times $T$ : (a) throughput of per GT, $T=100 \mathrm{~s}$; (b) throughput of per GT, $T=120 \mathrm{~s}$; (c) throughput of per GT, $T=140 \mathrm{~s}$; (d) throughput of per GT, $T=180 \mathrm{~s}$.

where

$$
\begin{aligned}
& \varphi_{j}[n]=\alpha_{k}^{j+1}[n] \log _{2}\left(1+\frac{P \gamma_{0}}{\left\|\mathbf{q}_{j}[n]-\mathbf{w}_{k}\right\|^{2}+H^{2}}\right), \\
& \psi_{j}[n]=\frac{1}{\ln 2} \cdot \frac{\alpha_{k}^{j+1}[n] P \gamma_{0}}{\left(\left\|\mathbf{q}_{j}[n]-\mathbf{w}_{k}\right\|^{2}+H^{2}\right)\left(\left\|\mathbf{q}_{j}[n]-\mathbf{w}_{k}\right\|^{2}+H^{2}+P \gamma_{0}\right)}, \quad \forall n .
\end{aligned}
$$




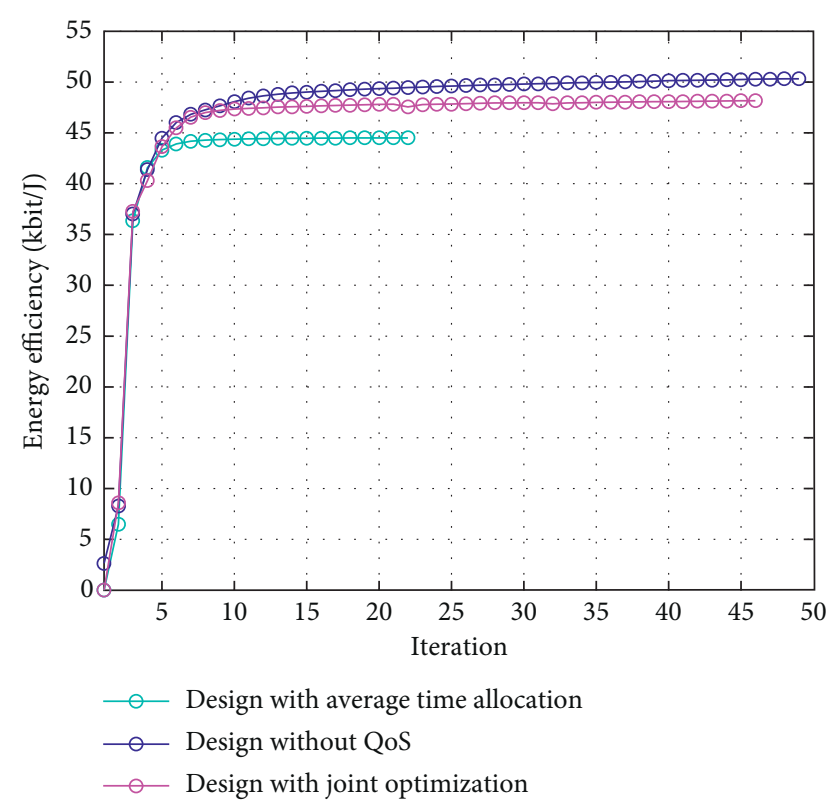

Figure 3: Convergence of the proposed Algorithm 1 for $T=140 \mathrm{~s}$.

As a result, with any given local point $\left\{\mathbf{q}_{j}[n]\right\}$, we can reformulate the optimization problem (14) as the following problem:

$$
\begin{gathered}
\max _{\left\{\mathbf{q}[n], \mathbf{v}[n], \kappa_{n}, \mathbf{a}[n]\right\}} \frac{R_{l b}(\mathbf{q}[n])}{P\left(\{\mathbf{q}[n]\},\left\{\kappa_{n}\right\}\right)}, \\
\text { s.t. }(10 \mathrm{a})-(10 f), \\
\kappa_{n} \geq 0, \quad \forall n, \\
\rho_{l b}(\{\mathbf{v}[n]\}) \geq \kappa_{n}^{2}, \quad \forall n, \\
\delta_{\mathrm{t}} \cdot R_{l b}(\{\mathbf{q}[n]\}) \geq Q_{\mathrm{m}}, \quad k \in \mathcal{N} .
\end{gathered}
$$

It is worth noting that the first-order Taylor expansions in (16) and (17) are tight at the given local point which means that problem (19) has the same objective value as that of problem (14). After problem (19), we can obtain the optimal trajectory $\left\{\mathbf{q}_{j+1}^{*}[n]\right\}$. In the next iteration, $\left\{\mathbf{q}_{j+1}^{*}[n]\right\}$ is used as the input for problem (13). In summary, the detail procedure is shown in Algorithm 1.

Note that problem (11) and problem (12) are special cases of problem (10). As a result, the proposed Algorithm 1 is also feasible to the special cases I and II.

\section{Numerical Results}

In this section, numerical results are presented to show the validation and effectiveness of the proposed designs. We assume that the altitude of the UAV is fixed at $H=100 \mathrm{~m}$. The number of GTs is $N=10$, and they are uniformly distributed within a $2 \mathrm{D}$ circular area with radius $0.8 \mathrm{~km}$. The QoS requirement for each GT is $Q_{\mathrm{m}}=30$ Mbits. We set the communication bandwidth $B=1 \mathrm{MHz}$, and the noise power spectral density is $N_{0}=-170 \mathrm{dBm} / \mathrm{Hz}$. The noise power is $\sigma^{2}=N_{0} B=-110 \mathrm{dBm}$. We also assume that the constant transmission power of each GT is $P=10 \mathrm{dBm}$, and the reference-received signal-to-noise ratio is $\gamma_{0}=80 \mathrm{~dB}$. Moreover, from [15], we set $c_{1}=9.26 \times 10^{-4}$ and $c_{2}=2250$. The threshold accuracy $\varepsilon$ in Algorithm 1 is set as $10^{-6}$. The initial and final locations of the UAV are assumed to be $\mathbf{q}[1]=\mathbf{q}[M]=[500,0]^{T}$. The maximum velocity and acceleration of the UAV are set as $V_{\max }=100 \mathrm{~m} / \mathrm{s}$ and $a_{\max }=5 \mathrm{~m} / \mathrm{s}^{2}$, respectively. The initial and final velocities are assumed as $\mathbf{v}[1]=\mathbf{v}[M]=[0,30]^{T}$.

Figure 2 shows the throughput of each GT for different period times. We can see that the change of throughput per GT is closely related to the period time and location. For example, for the 10th GT, its throughput rapidly increases from $T=100 \mathrm{~s}$ to $T=120 \mathrm{~s}$, which means that the UAV can achieve a better channel to communicate with the 10th GT in the later horizon time leading to a higher transmission rate. Note that, for the three designs, the throughput of each GT is not monotonically increasing with regard to the horizon time. In fact, the period time can affect the UAV's trajectory, and then, it has an effect on the channel conditions, which impacts the GT scheduling. On the other hand, if the UAV flies without considering QoS requirements (i.e., the special case II), it can be observed that the throughput of the 3rd and 7th GTs are below the QoS threshold, as shown in Figures $2(a)-2(c)$. What is worse, the throughput of the 3rd GT actually is zero in Figures 2(a)-2(c), which means that the 3rd GT is not allocated any slot to transmit data. Hence, in the special case II, the UAV cannot ensure the minimum communication requirement for each GT, which may result in a larger gap between maximum and minimum throughput. The special case II causes a severe limitation on the communication scenarios requiring users QoS. As for the design with average time allocation (i.e., the special case I), each GT is allocated an equal number of time slots for uploading data to the UAV. As a result, these GTs' throughputs are relatively regular and even. However, the optimal EE of this case actually is the lowest, as shown in Figure 3.

Figure 3 shows the convergence performance of the proposed Algorithm 1. In this result, we compare three design schemes for $T=140 \mathrm{~s}$. It can be observed that the energy efficiency increases rapidly with the number of iterations at the beginning and then rises slowly until converges to the prescribed accuracy. In addition, it is observed that the UAV can achieve higher EE when it flies without considering QoS requirements, and the UAV's EE is minimum when it adopts the method of average time allocation, which is in line with our expectations.

Figure 4 shows the three cases of optimal energy-efficient trajectory obtained by Algorithm 1 for several different period times $T$. We compare the jointly optimal trajectory of the UAV with the special cases I and II. It can be observed that the disparity of the trajectory between jointly optimal design and special case II becomes smaller as the period time $T$ increases. That is because there are more time slots for the UAV to utilize with the increasing period time. Thus, the ratio of the time satisfying QoS requirements to the horizon time $T$ becomes increasingly small, which means that, in the great majority of time slots, the GT 
(1) Initialize $\left\{\mathbf{q}_{j}[n], \mathbf{v}_{j}[n]\right\}$. Let $j=0$, accuracy $\varepsilon>0$.

(3) Solve problem (13) for the given $\left\{\mathbf{q}_{j}[n]\right\}$, and denote the optimal solution as $\left\{\alpha_{k}^{j+1 *}[n]\right\}$.

(4) Update $\alpha_{k}^{j+1}[n]=\alpha_{k}^{j+1 *}[n]$.

(5) Solve problem (19) for the given $\left\{\mathbf{q}_{j}[n], \mathbf{v}_{j}[n], \alpha_{k}^{j+1}[n]\right\}$, and denote the optimal solution as $\left\{\mathbf{q}_{j+1}^{*}[n], \mathbf{v}_{j+1}^{*}[n]\right\}$.

(6) Update $\mathbf{q}_{j+1}[n]=\mathbf{q}_{j+1}^{*}[n], \mathbf{v}_{j+1}[n]=\mathbf{v}_{j+1}^{*}[n]$.

(7) Update $j=j+1$.

(8) Until converges to the prescribed accuracy $\varepsilon$.

Algorithm 1: Alternative iteration method for problem (10).
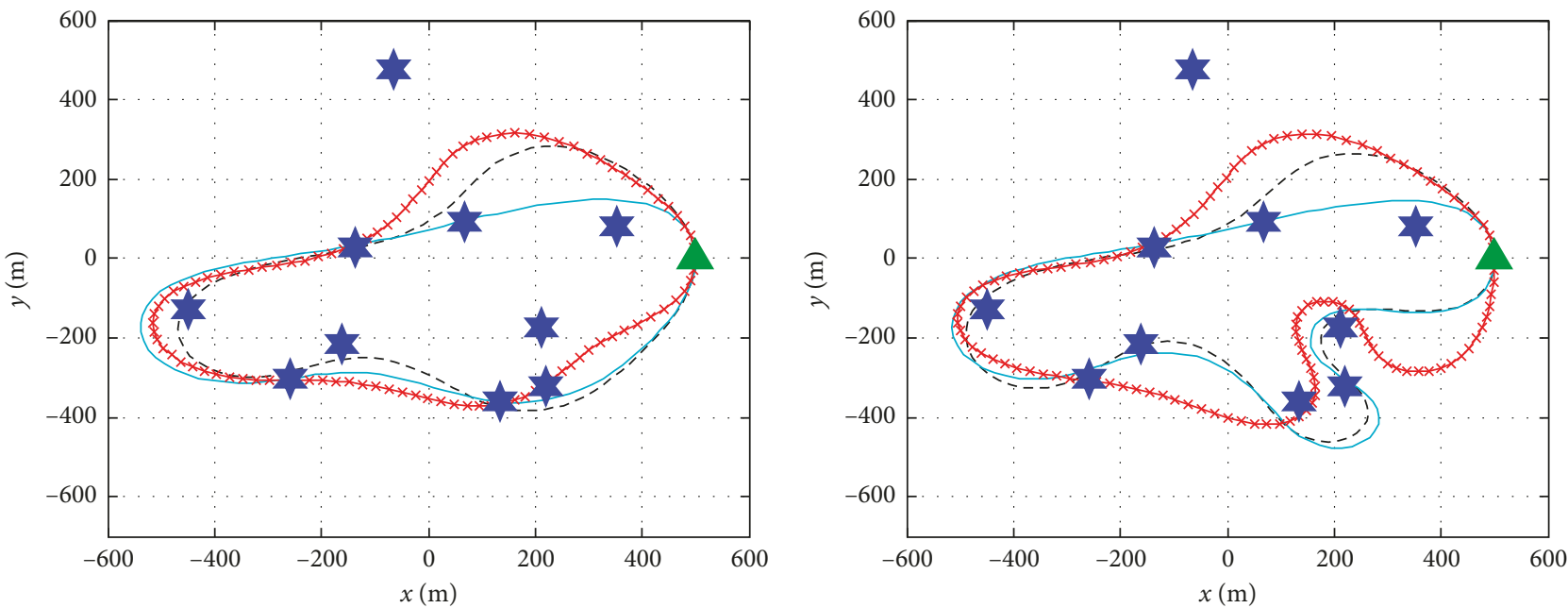

- - - EE-max with average time allocation

_ EE-max without QoS

$\rightarrow$ EE-max with joint optimization

(a)

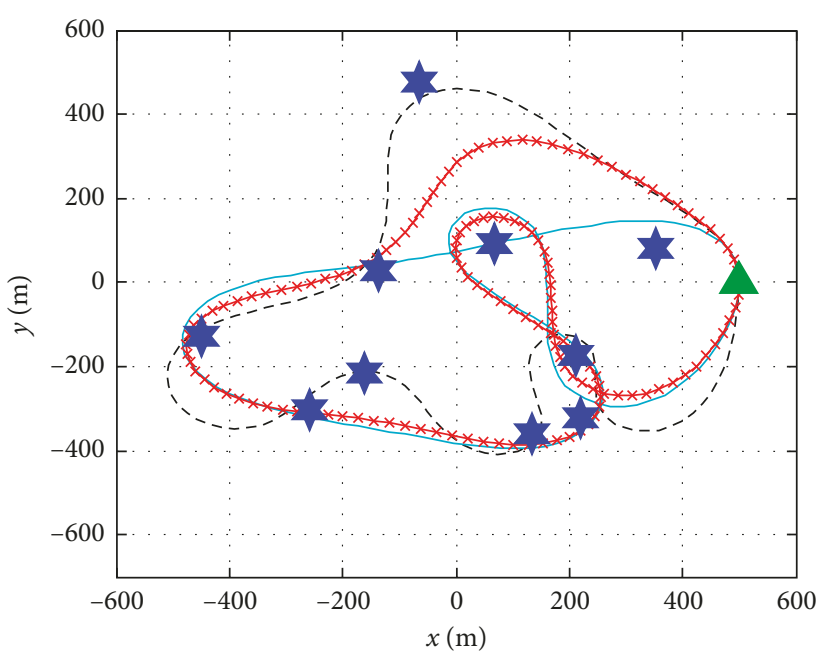

- - EE-max with average time allocation

— EE-max without QoS

$*$ EE-max with joint optimization

(c)

\section{- - - EE-max with average time allocation \\ _ EE-max without QoS \\ $\star$ EE-max with joint optimization}

(b)

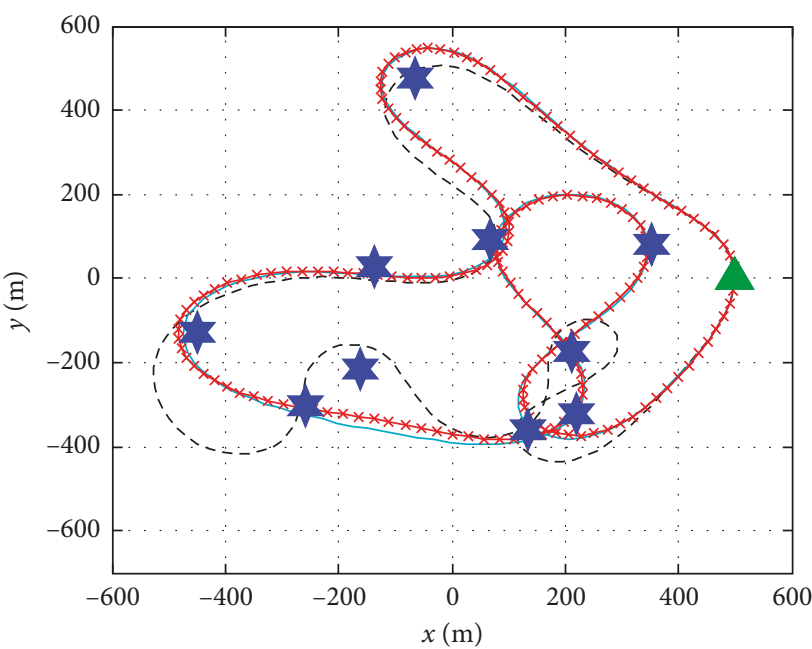

- - EE-max with average time allocation

_ EE-max without QoS

$*$ EE-max with joint optimization

(d)

FIgURE 4: Comparison of optimal energy-efficient trajectory of the UAV with different period times $T$. The initial and final locations are marked by " ," and the locations of GTs are marked by “ $\downarrow$." (a) Optimal trajectory, $T=100$ s. (b) Optimal trajectory, $T=120 \mathrm{~s}$. (c) Optimal trajectory, $T=40$ s. (d) Optimal trajectory, $T=180 \mathrm{~s}$. 
TABle 1: Performance comparison for the two special cases with the proposed jointly optimal design.

\begin{tabular}{|c|c|c|c|c|c|c|}
\hline \multirow[b]{2}{*}{ Period time (s) } & \multicolumn{3}{|c|}{ Energy efficiency (kbits/J) } & \multicolumn{3}{|c|}{ Average rate (Mbps) } \\
\hline & $\begin{array}{l}\text { Average time } \\
\text { allocation }\end{array}$ & $\begin{array}{c}\text { Number of QoS } \\
\text { requirements }\end{array}$ & $\begin{array}{c}\text { Joint } \\
\text { optimization }\end{array}$ & $\begin{array}{l}\text { Average time } \\
\text { allocation }\end{array}$ & $\begin{array}{l}\text { Number of QoS } \\
\text { requirements }\end{array}$ & $\begin{array}{c}\text { Joint } \\
\text { optimization }\end{array}$ \\
\hline 100 & 47.95 & 51.51 & 49.06 & 5.34 & 5.65 & 5.43 \\
\hline 110 & 46.53 & 50.16 & 46.8 & 5.44 & 5.61 & 5.51 \\
\hline 120 & 45.59 & 48.63 & 45.73 & 5.43 & 5.75 & 5.44 \\
\hline 130 & 44.27 & 50.57 & 46 & 5.38 & 5.79 & 5.5 \\
\hline 140 & 44.5 & 50.5 & 48.38 & 5.43 & 5.77 & 5.56 \\
\hline 150 & 44.76 & 50.31 & 48.59 & 5.46 & 5.74 & 5.6 \\
\hline 160 & 47.59 & 48.8 & 48.67 & 5.57 & 5.62 & 5.61 \\
\hline 170 & 45.31 & 49.32 & 49.23 & 5.57 & 5.64 & 5.62 \\
\hline 180 & 45.53 & 49.56 & 49.38 & 5.57 & 5.68 & 5.67 \\
\hline
\end{tabular}

scheduling of these two designs is the same. As for the special case I, due to employing the average time allocation technique, the UAV is more likely to fly closer to each GT, especially when $T$ is not large; this leads to the special case I having a better fairness compared with the other two designs, as shown in Figure 2.

Table 1, respectively, gives the comparison of the maximum energy efficiency and total average rate of the system achieved by the three energy-efficient trajectory designs in different periods $T$. It is observed that the EE of the design with average time allocation is invariably lowest as the average time allocation severely hinders the algorithm's optimization performance. For the design without QoS requirements, the UAV's EE is always largest in different period times $T$. The main reason is that no QoS requirements give the UAV more freedom to optimize its EE in each iteration. From this table, the performance of the joint optimization design falls in between, but it overcomes the main defects of the other two designs. It is interesting to note that, for a small period time (e.g., $T=100 \mathrm{~s}$ to $120 \mathrm{~s}$ ), the energy-efficient performance of the joint optimization design is close to the design with average time allocation because there is little time freedom for the UAV to optimize the trajectory. Correspondingly, for a large period time (e.g., $T=160 \mathrm{~s}$ to $180 \mathrm{~s}$ ), the UAV has sufficient time slots to optimize its trajectory, while the effect of the QoS constraint is inconspicuous. As a result, for a large period time, the design with joint optimization and the design without QoS requirements have the similar EE values and trajectory, as shown in Figure $4(\mathrm{~d})$. For the period time within $120 \leq T \leq 160$, the jointly optimal design has more time freedom to maximize the EE values compared to the design with average time allocation, while the QoS constraint has a nonnegligible effect on the EE maximization compared to the design without QoS requirements. In this sense, the jointly optimal design actually strikes a trade-off between the special cases I and II.

In summary, the GT scheduling of the joint optimization design is more flexible compared to the special case I, and it leads to better performance. Although the EE of the joint optimization design is smaller compared to the case II, it can ensure the QoS requirements for all GTs, which is more in line with actual requirements and hence has more practical significance.
From Table 1, we also know that the EE of the UAV is not monotonically increasing with respect to the period time $T$; this gives us the freedom to find the optimal period time that can further improve the UAV's EE on the basis of this paper. This problem will be left to our future work.

\section{Conclusions}

In this paper, we investigate the energy efficiency of a UAV wireless communication system with multiple GTs. By deriving the propulsion energy consumption and communication rate models of the UAV, we establish the energy efficiency framework that jointly optimizes the scheduling of GTs and the UAV's trajectory. In addition, we establish two special cases as benchmarks to illustrate the optimal performance of our objective problem. To solve the formulated problem, we propose an iterative algorithm that jointly applies standard LP and successive convex optimization techniques. Using this algorithm, a locally optimal solution is obtained. Numerical results show that the energy efficiency of the UAV can be enhanced significantly with our design and that optimal performance is achieved compared with the other two benchmark designs, which is consistent with our expectations.

\section{Conflicts of Interest}

The authors declare that there are no conflicts of interest regarding the publication of this paper.

\section{Acknowledgments}

This work was supported in part by the National Natural Science Foundation of China (61703197, 61561032, and 61461029), China/Jiangxi Postdoctoral Science Foundation Funded Project (2014MT561879 and 2014KY046), Young Scientists Project Funding of Jiangxi Province (20162BCB23010 and 20153BCB23020), and the Natural Science Foundation of Jiangxi Province (20114ACE00200).

\section{References}

[1] Y. Zeng, R. Zhang, and T. J. Lim, "Wireless communications with unmanned aerial vehicles: opportunities and challenges," 
IEEE Communications Magazine, vol. 54, no. 5, pp. 36-42, 2016.

[2] J. Valente, D. Sanz, A. Barrientos, J. D. Cerro, A. Ribeiro, and C. Rossi, "An air-ground wireless sensor network for crop monitoring," Sensors, vol. 11, no. 6, pp. 6088-6108, 2011.

[3] S. Waharte and N. Trigoni, "Supporting search and rescue operations with UAVs," in Proceedings of the 2010 International Conference on Emerging Security Technologies (EST), pp. 142-147, Canterbury, UK, September 2010.

[4] M. Abdelkader, M. Shaqura, M. Ghommem, N. Collier, V. Calo, and C. Claudel, "Optimal multi-agent path planning for fast inverse modeling in UAV-based flood sensing applications," in Proceedings of the 2014 International Conference on Unmanned Aircraft Systems (ICUAS), pp. 64-71, Orlando, FL, USA, May 2014.

[5] I. Maza, F. Caballero, J. Capitán, J. Martínez-de Dios, and A. Ollero, "Experimental results in multi-UAV coordination for disaster management and civil security applications," Journal of Intelligent \& Robotic Systems, vol. 61, no. 1-4, pp. 563-585, 2011.

[6] C. Wu, X. Cao, R. Lin, and F. Wang, "Registration-based moving vehicle detection for low-altitude urban traffic surveillance," in Proceedings of the 8th World Congress on Intelligent Control and Automation (WCICA 2010), pp. 373-378, Jinan, China, July 2010.

[7] Y. Zeng, R. Zhang, and T. J. Lim, "Throughput maximization for UAV-enabled mobile relaying systems," IEEE Trans. Commun., vol. 64, no. 12, pp. 4983-4996, Dec. 2016.

[8] Facebook, Connecting the World from the Sky, Technical Report, Facebook, Inc., Menlo Park, CA, USA, 2014.

[9] S. Katikala, "Google project loon," InSight: Rivier Academic Journal, vol. 10, no. 2, 2014.

[10] M. Mozaffari, W. Saad, M. Bennis, and M. Debbah, "Drone small cells in the clouds: design, deployment and performance analysis," in Proceedings of the IEEE Global Communications Conference (GLOBECOM 2015), pp. 1-6, San Diego, CA, USA, December 2015.

[11] M. Mozaffari, W. Saad, M. Bennis, and M. Debbah, "Unmanned aerial vehicle with underlaid device-to-device communications: performance and tradeoffs," IEEE Transactions on Wireless Communications, vol. 15, no. 6, pp. 3949-3963, 2016.

[12] J. Lyu, Y. Zeng, R. Zhang, and T. J. Lim, "Placement optimization of UAV-mounted mobile base stations," IEEE Communications Letters, vol. 21, no. 3, pp. 604-607, 2017.

[13] A. Merwaday and I. Guvenc, "UAV assisted heterogeneous networks for public safety communications," in Proceedings of the IEEE Wireless Communications and Networking Conference, pp. 329-334, New Orleans, LA, USA, March 2015.

[14] M. M. Azari, F. Rosas, and K.-C. Chen, "Optimal UAV positioning for terrestrial-aerial communication in presence of fading," in Proceedings of the IEEE Global Communications Conference (GLOBECOM 2016), pp. 1-7, Washington, DC, USA, December 2016.

[15] Y. Zeng and R. Zhang, "Energy-efficient UAV communication with trajectory optimization," IEEE Transactions on Wireless Communications, vol. 16, no. 6, pp. 3747-3760, 2017.

[16] Y. Chen, S. Zhang, S. Xu, and G. Y. Li, "Fundamental tradeoffs on green wireless networks," IEEE Communications Magazine, vol. 49, no. 6, pp. 30-37, 2011.

[17] I. Bor-Yaliniz, A. El-Keyi, and H. Yanikomeroglu, "Efficient 3-D placement of an aerial base station in next generation cellular networks," in Proceedings of the IEEE International Conference on Communications (ICC 2016), Kuala Lumpur, Malaysia, May 2016.
[18] M. Mozaffari, W. Saad, M. Bennis, and M. Debbah, "Efficient deployment of multiple unmanned aerial vehicles for optimal wireless coverage," IEEE Communications Letters, vol. 20, no. 8, pp. 1647-1650, 2016.

[19] M. Alzenad, A. El-keyi, F. Lagum, and H. Yanikomeroglu, "3D placement of an unmanned aerial vehicle base station (UAVBS) for energy-efficient maximal coverage," IEEE Wireless Communications Letters, vol. 6, no. 4, pp. 434-437, 2017.

[20] J. W. Zhang, Y. Zeng, and R. Zhang, "Spectrum and energy efficiency maximization in UAV-enabled mobile relaying," in Proceedings of the IEEE International Conference on Communications (ICC 2017), Paris, France, May 2017.

[21] C. Zhan, Y. Zeng, and R. Zhang, "Energy-efficient data collection in UAV enabled wireless sensor network," IEEE Wireless Communications Letters, 2017, https://arxiv. org/abs/1708.00221.

[22] U. Mengali and A. N. D’Andrea, Synchronization Techniques for Digital Receivers, Springer, Berlin, Germany, 1997. 


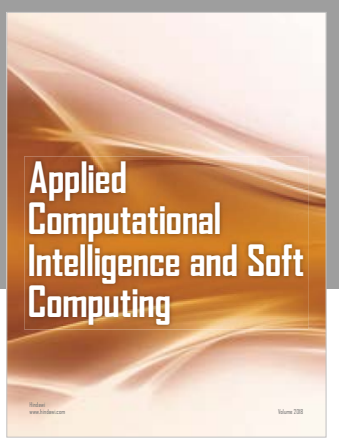

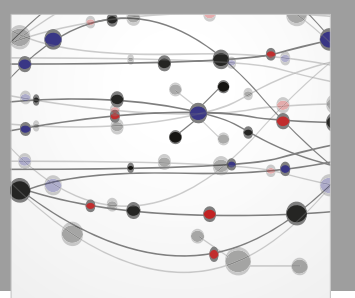

The Scientific World Journal
Submit your manuscripts at

Computing
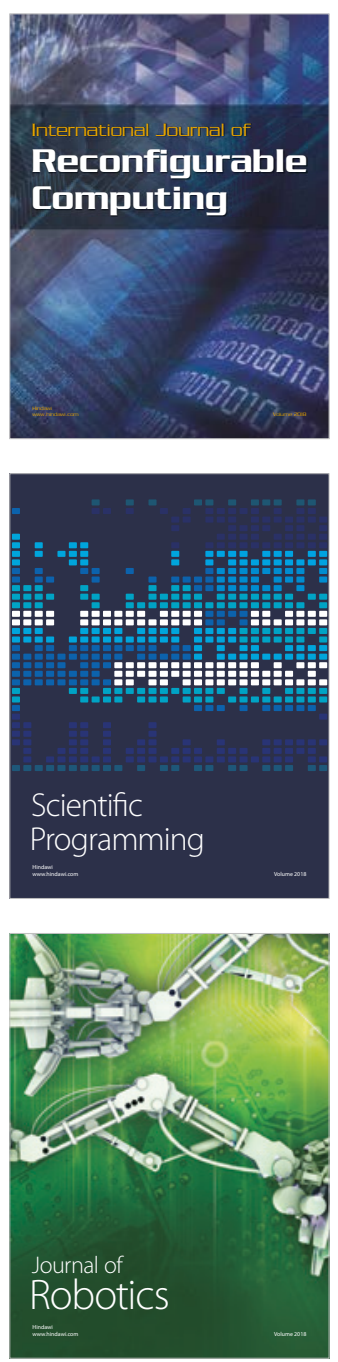

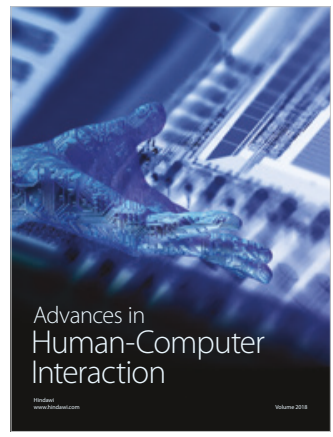

Human-Compute

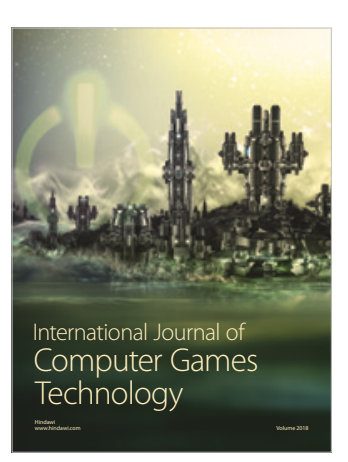

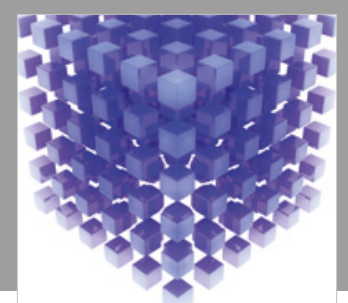

Mathematical Problems in Engineering

\section{Engincering}
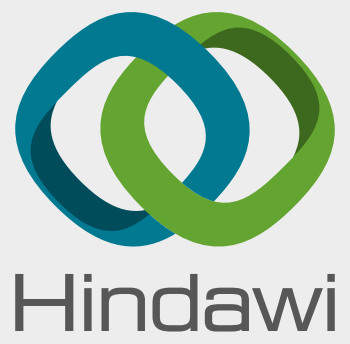

www.hindawi.com
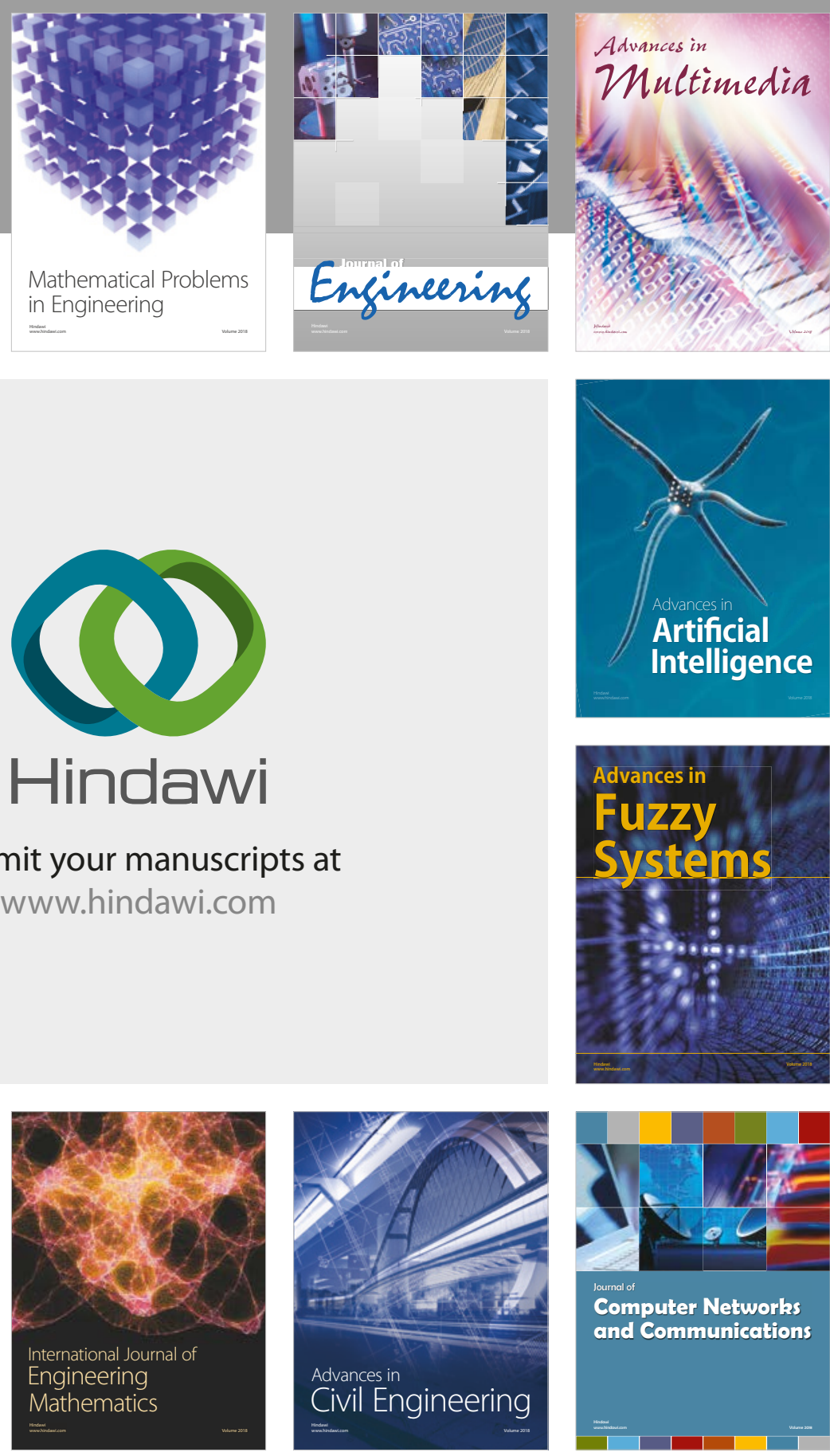

Computer Networks and Communications

Multimedia
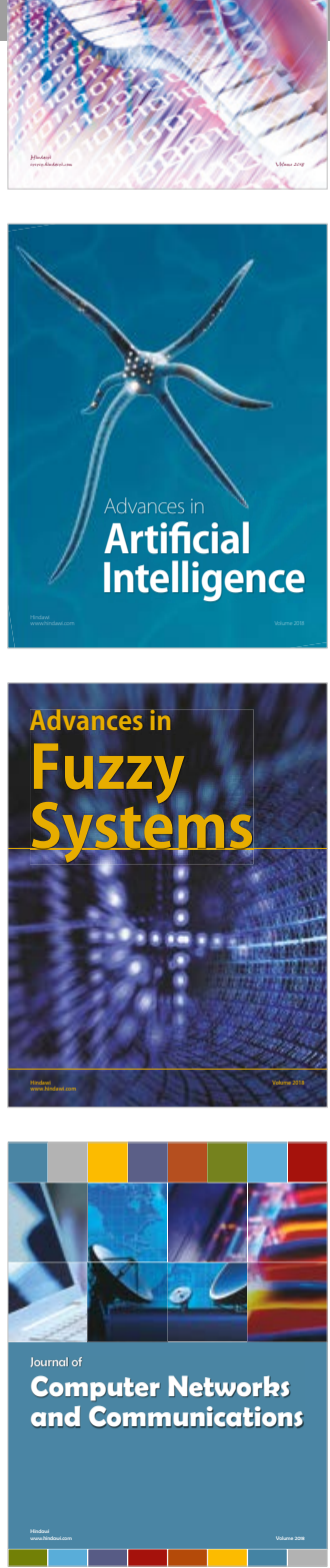

Advances in

Modelling \&

Simulation

in Engineering

interaction

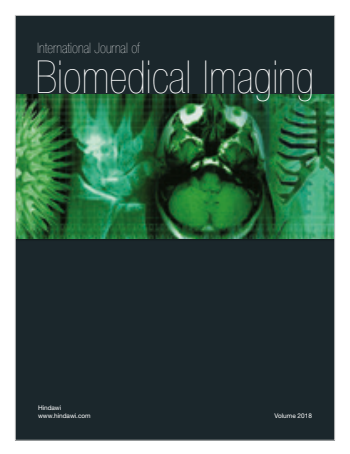

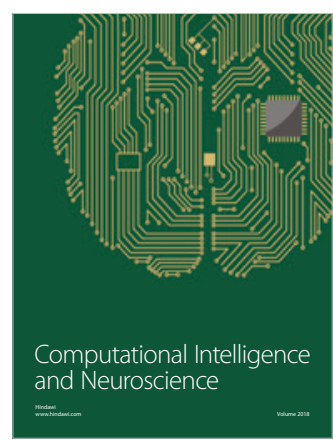

